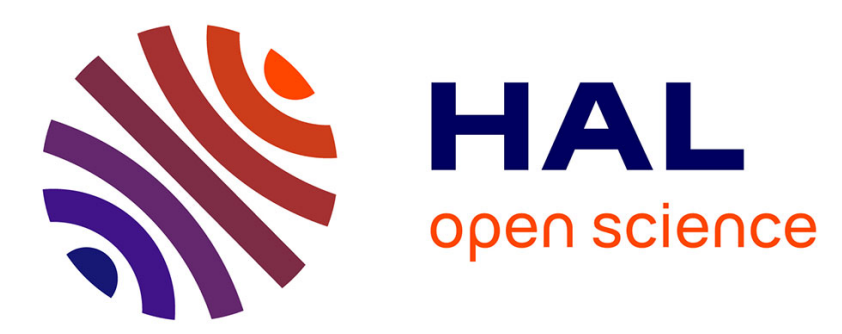

\title{
Les expériences réputées psychotiques dans la population générale: essai de problématisation \\ Renaud Evrard
}

\section{To cite this version:}

Renaud Evrard. Les expériences réputées psychotiques dans la population générale: essai de problématisation. Annales Médico-Psychologiques, Revue Psychiatrique, 2011, 169 (5), pp.282-287. 10.1016/j.amp.2010.01.008 . halshs-02137277

\section{HAL Id: halshs-02137277 \\ https://shs.hal.science/halshs-02137277}

Submitted on 17 Jul 2019

HAL is a multi-disciplinary open access archive for the deposit and dissemination of scientific research documents, whether they are published or not. The documents may come from teaching and research institutions in France or abroad, or from public or private research centers.
L'archive ouverte pluridisciplinaire HAL, est destinée au dépôt et à la diffusion de documents scientifiques de niveau recherche, publiés ou non, émanant des établissements d'enseignement et de recherche français ou étrangers, des laboratoires publics ou privés. 


\section{Renaud EVRARD}

\section{Les expériences réputées psychotiques dans la population générale : essai de problématisation}

Résumé :

Des études épidémiologiques et psychométriques récentes ont mis en évidence la présence sous-estimée d'hallucinations et de délires chez de nombreux individus n'ayant pas reçu le diagnostic de psychose. Comment les modèles psychologiques rendent-ils compte de ce «continuum psychotique»? Cet article décrit les éléments clefs d'un débat principalement anglo-saxon en tentant d'y introduire un point de vue critique qui prend sa source dans une psychopathologie psychanalytique. Cette critique porte notamment sur le type de mesures effectuées qui, pour la plupart, passent par des questionnaires avec des items venant fixer dans le registre de la psychose certaines croyances ou certains vécus, repositionnant le discours psychiatrique face à la «bizarrerie» de certaines «expériences exceptionnelles ». Après avoir présenté une alternative méthodologique basée sur des entretiens diagnostiques semistructurés et des critères différentiels issus de la psychanalyse, nous interrogerons les enjeux de cette évaluation de la psychose.

Mots-clefs : prévalence des troubles mentaux dans la population générale - continuum psychotique - expériences exceptionnelles - diagnostic différentiel - modèles psychologiques de la psychose

\section{Psychosis-like experiences in non-clinical population: review of some issues}

Abstract:

Epidemiological and psychometric studies have recently highlighted the underestimated prevalence of hallucinations and delusions in many individuals who have never received the diagnostic of psychosis. How psychological models account for this "continuum of psychosis"? This article describes the key elements of a mainly Anglo-Saxon debate, attempting to introduce a critical perspective that is rooted in a psychoanalytic psychopathology. This critical review will applied in particular on measurements which, for the most part, are based on questionnaires with items setting into psychosis' area some beliefs and experiences, repositioning the psychiatric discourse on the "peculiarity" of some "exceptional experiences". After presenting an alternative methodology based on semi-structured diagnostic interviews and differential criteria from psychoanalysis, we will examine the issues involved in this assessment of psychosis.

Key-words : prevalence of mental disorders in non-clinical population - psychosis continuum - exceptional experiences - differential diagnosis - psychological models of psychosis 
Hallucinations et psychose

Autant chez le public que chez les professionnels de santé, une présomption persistante consiste à dire que les hallucinations et les délires sont les produits de troubles psychiatriques qui n'ont rien à voir avec la cognition normale. Cette présomption a eu comme effet que la plupart des recherches sur ces questions se sont focalisées sur des groupes de patients psychiatriques déterminés à partir de critères diagnostiques généraux comme relevant de maladies telles que la schizophrénie ou la psychose maniaco-dépressive. Or, les recherches en psychiatrie d'avant 1920 (environ) avaient une orientation différente, s'intéressant davantage aux «phénomènes psychologiques » qu'aux diagnostics généraux de maladies mentales, voie qui semble revenue au premier plan depuis 1980 [5]. Il suffit de penser à la fonction que Freud donne à l'hallucinatoire dans l'éveil de la vie psychique et la réalisation de désir [12,18]. Les résultats de ces recherches ont apporté une nouvelle lumière sur les processus psychologiques et neurophysiologiques à la base des hallucinations et des délires, tout en remettant en cause la frontière entre maladie mentale et vie mentale normale. Notre article vise à confronter, d'une part, des données sur les expériences psychotiques et des interprétations émanant de la littérature anglo-saxonne des 25 dernières années et, d'autre part, une psychopathologie d'orientation psychanalytique intégrant certains apports de Lacan.

La fin du XIXe siècle fut marquée par le courant de l'hypnotisme et l'étude de l'hystérie. La mise en évidence d'hallucinations, de divisions de la personnalité et d'autres phénomènes psychologiques associés permirent les avancées les plus importantes de la psychologie et de la psychiatrie dynamique de cette époque. Or, la majorité des sujets impliqués dans ces travaux n'étaient pas considérés comme relevant de la psychose.

Dans les premières années du $\mathrm{XX}^{\mathrm{e}}$ siècle, les hallucinations ont commencé à être considérées principalement comme des symptômes de la schizophrénie [44]. Pour Eugen Bleuler [8], qui a forgé le terme schizophrénie préférentiellement à la précédente désignation de dementia praecox de Kraepelin, les hallucinations étaient des symptômes accessoires de la maladie. Kurt Schneider [45] a plus tard défendu l'idée que certains types d'hallucination de modalité auditive devraient être considérés comme des symptômes « de premier rang » de la schizophrénie, un point de vue qui se reflète dans les critères diagnostiques modernes tels que ceux que l'on peut trouver dans le Diagnostic and Statistical Manual of Mental Disorders (DSM [2]) à partir de sa troisième édition.

Le retour à une conception des hallucinations en tant que «phénomènes psychologiques » indépendants des considérations psychopathologiques peut se lire dans la définition qu'en donnent Slade et Bentall ([49], p. 23) : « Toute expérience semblable à une perception qui (a) survient en l'absence d'un stimulus approprié, (b) possède la pleine force ou l'impact de la vraie perception correspondante, et (c) n'est pas soumise au contrôle volontaire ou direct de celui qui la vit». Une telle définition de l'expérience hallucinatoire a servi de support aux études épidémiologiques et psychométriques des hallucinations dans la population générale, dont les données posent d'importants problèmes aux modèles de la psychose faisant des hallucinations leur pierre angulaire.

Les données du « continuum psychotique »

De nombreuses études sur des personnes n'ayant pas reçu de diagnostic psychiatrique décèlent la présence de vécus diversement nommés : anomalous experiences, psychosis-like experiences, croyances et expériences paranormales ou encore expériences exceptionnelles [56, 9, 46]. Ces vécus sont classés parmi les hallucinations et les délires habituellement attribués à la psychose, mais pouvant apparaître sous des formes atténuées. Pour une proportion significative de ces individus du tout-venant, ce sont les seuls critères de troubles mentaux détectés [57]. Cela suggère que les croyances et expériences inhabituelles ne seraient pas que du domaine de la psychose déclarée, le groupe des schizophrénies devant être vu comme une extrémité d'un continuum de la psychose [56, 51].

L'hypothèse d'un continuum a renforcé en retour l'attention des chercheurs sur ces expériences d'hallucinations et de délires (que nous appellerons brièvement « expériences psychotiques ») chez des personnes qui, par ailleurs, semblaient «normales ». Cet intérêt accru possède deux versants : il permet tout d'abord d'affiner la description de processus psychotiques sous-estimés chez tout à chacun. L'exploration des facteurs de risque modulant ces expressions quasipsychotiques permettrait de mieux comprendre la psychose que la recherche restreinte à des individus à l'extrême fin de la distribution de la dimension psychotique [57]. Mais, d'un autre côté, il laisse suspecter que beaucoup de «populations à risque de psychose » échapperaient encore au circuit médico-psychologique. Il y aurait des expressions de la psychose suffisamment viables pour n'être pas encore détectées par les dispositifs actuels [54]. Des stratégies d'intervention 
nouvelles et possiblement iatrogènes sont proposées pour ces personnes qui sortaient jusque-là des tableaux psychiatriques, réveillant des enjeux idéologiques, politiques et économiques de médicalisation d'un vécu intime [21].

Les observations d'un continuum entre les expériences psychotiques et les états normaux soulèvent la question de savoir si on doit toujours considérer ces expériences comme pathologiques. Sans aucun doute hallucinations et délires apparaissent-ils souvent dans le contexte de troubles mentaux ou physiques graves. Toutefois, ces données sont contrebalancées par celles donnant une sorte de «taux de base » des expériences psychotiques dans la population générale.

La première tentative systématique de déterminer si les hallucinations pouvaient survenir chez les personnes ne souffrant pas de maladie mentale ou physique fut réalisée en Grande-Bretagne à la fin du XIX ${ }^{\mathrm{e}}$ siècle par la Society for Psychical Research (SPR) de Londres ${ }^{1}$. Elle avait été précédée par une enquête à grande échelle sur les « hallucinations télépathiques », c'est-à-dire les hallucinations coïncidant avec des événements qui se révéleront par la suite bien réels [22, 29]. Dans l'étude connue sous le nom de Census of hallucinations [48], un total de 7717 hommes et 7599 femmes répondirent à cette question : "Avez-vous déjà eu l'impression vivace de voir, ou d'être touché, ou d'entendre une voix ; impression qui, autant que vous puissiez le découvrir, n'était due à aucune cause extérieure ?»

Bien que les parapsychologues de la SPR n'aient pas tenté d'obtenir un échantillonnage vraiment aléatoire, ils ont exclu de l'étude toute personne qui montrait des signes évidents de maladie mentale ou physique. Au sein de l'échantillon total, 7,8\% des hommes et $12 \%$ des femmes ont signalé au moins une expérience hallucinatoire ayant l'aspect de la réalité, au contenu parfois religieux ou paranormal, le type le plus commun étant l'hallucination visuelle d'une personne vivante qui n'était pas présente au moment de l'expérience.

Au final, le Census of hallucinations obtint des réponses affirmatives auprès d'environ 1 personne sur 10. Il est très étonnant de voir que les résultats de cette étude ont résisté à l'épreuve du temps. La première enquête psychiatrique moderne sur les hallucinations fut dirigée par McKellar [35], qui trouva que 125 personnes «normales » sur les 500 interrogées avaient signalé avoir vécu au moins une expérience hallucinatoire. Posey et Losch [40] ont interrogé un échantillon de 375 étudiants, et ont déclaré que $39 \%$ disaient avoir eu le symptôme schneiderien principal qui consiste à entendre une voix tout haut, et que $5 \%$ déclaraient tenir des conversations avec leurs hallucinations. Des enquêtes ultérieures sur les étudiants au Royaume-Uni [6] et aux États-Unis [3] ont également révélé qu'une proportion surprenante d'entre eux signalait des antécédents d'expériences hallucinatoires.

L'enquête la plus complète à ce jour a été exposée par Tien [52] à partir d'une vaste étude par questionnaires de l'Epidemiological Catchment Area (ECA) sur les symptômes psychiatriques dans un échantillon aléatoire de la population générale. Au total, 18572 personnes furent évaluées, et 15258 d'entre elles acceptèrent d'être réévaluées un an plus tard. Tien a comparé les données de cette étude avec celles de Sidgwick et al. [48] collectées presque un siècle plus tôt. Les données des deux études se sont révélées remarquablement similaires. Les estimations de la prévalence des hallucinations au cours d'une vie dans l'étude ECA était de $13,0 \%$ dans la première évaluation et de $11,1 \%$ dans la seconde, chiffres qui ne diffèrent pas significativement des résultats de Sidgwick et al. Une enquête plus récente sur les expériences psychotiques réalisée plus précisément sur la population britannique confirme également les chiffres de la SPR [26].

Les parapsychologues furent donc des contributeurs actifs dans ces recherches sur les hallucinations dans la population générale. Ils cherchaient à la fois à légitimer l'intérêt qu'ils portaient aux témoignages de phénomènes paranormaux et à mieux comprendre leurs caractéristiques, afin que les vécus spontanés guident les expérimentations contrôlées. Ils montrèrent également que ces expériences pouvaient avoir des répercussions positives comme négatives. Auparavant, les croyances au paranormal et les expériences paranormales étaient majoritairement évaluées dans leur rapport à la pathologie, aux déficits cognitifs, bref aux corrélatifs négatifs [24]. Cette orientation a longtemps semblé en consonance avec nos autres connaissances sur la psychose, les hallucinations et les délires. Les parapsychologues furent les plus enclins à réaliser des mesures de corrélats positifs des « expériences vécues comme paranormales » (ou « expériences exceptionnelles » selon la terminologie plus neutre en vigueur ; [4]), en se concentrant d'abord sur les expériences de hors corps [33] mettant en évidence que ces expériences pouvaient contribuer à une meilleure santé mentale. L'étude des expériences psychotiques dans la population générale est une manière de traiter les problèmes posés par la

1 La Society for Psychical Research regroupait des personnalités morales importantes (comme l'ancien premier ministre Arthur Balfour ou le professeur respecté Henry Sidgwick) ainsi que des scientifiques reconnus (Oliver Lodge, William Crookes, Lord Rayleigh, etc.) et des pionniers de la psychologie (William James, F.W.H. Myers, Charles Richet, Pierre Janet, Henri Bergson, Sigmund Freud, Carl G. Jung, etc.) (cf. [20, 29]). L'objectif de la société était de mener des recherches scientifiques sur les phénomènes dits paranormaux, ce qui est connu de nos jours sous le vocable « parapsychologie ». Elle poursuit toujours ses activités. 
prévalence élevée et l'ambivalence des expériences exceptionnelles.

Répercussions négatives et positives

Beaucoup de patients schizophrènes relatent des hallucinations et des délires dont ils peuvent souffrir, notamment du fait de la stigmatisation et de la marginalisation qui accompagnent l'aveu de tels vécus. Or, les études sur les évolutions à long terme des patients diagnostiqués comme schizophrènes indiquent, dans l'ensemble, qu'à peu près un tiers d'entre eux guérissent complètement de leurs expériences psychotiques, environ un tiers restent perturbés et handicapés tout au long de leur vie adulte, et un tiers ont un résultat de type intermédiaire [8, 14]. Encore peu d'études s'intéressent à ce que ces rémissions de schizophrénie viennent signifier [53].

Cependant, il est important de noter que ces données ne sont pas généralisables à toutes les personnes qui font l'expérience des hallucinations et des délires, parce que les patients inclus dans les études longitudinales ont souvent reçu un diagnostic formel de psychose et souffrent forcément de multiples symptômes et de difficultés sociales. De sorte que ces études ne nous apprennent presque rien sur l'histoire naturelle des expériences psychotiques et leurs conséquences spécifiques sur l'individu. Nous allons prendre deux exemples - celui des « entendeurs de voix » et celui des hallucinations non-psychotiques des enfants et des adolescents - montrant que ces expériences peuvent avoir à la fois des répercussions positives et négatives sur les individus qui les vivent, ce qu'il est important de prendre en compte avant tout traitement possible de leurs demandes.

Les « entendeurs de voix »

Les traités de psychiatrie affirment qu'entendre des voix est un symptôme de psychopathologie, et que prêter attention au contenu de ces voix déconnecte de la réalité. Mais est-ce valable pour tout le monde ? Même des patients hallucinés chroniques peuvent qualifier leurs voix de plaisantes et les intégrer à leur identité et à leur réseau social [36]. Les études épidémiologiques [43, 6, 55, 3] montrent que 2 à $4 \%$ de la population générale entend des voix et qu'environ un tiers seulement de ces personnes sont amenées à chercher de l'aide. Pourquoi les autres ne cherchent-ils pas d'aide pour un phénomène habituellement décrit comme un symptôme ?

Les psychiatres néerlandais Romme et Escher ont recruté des personnes ayant des hallucinations auditives - rebaptisés 《entendeurs de voix 》- dans la population générale. Ils ont soutenu qu'il existait un processus d'adaptation aux hallucinations [41]. Les nombreuses stratégies d'ajustement (de coping) mises en place étaient souvent personnalisées, mais les personnes traversaient les mêmes phases (de surprise, d'organisation puis de stabilisation). Approximativement les deux tiers des personnes interrogées se décrivaient comme ayant des difficultés pour s'en sortir avec leurs voix, tandis qu'un tiers se décrivait comme les supportant bien [41].

$70 \%$ des adultes disaient avoir commencé à entendre des voix suite à un « événement traumatique » (mort d'un membre de la famille, divorce, déménagement, abus sexuel, etc.), lequel avait souvent eu lieu dans l'enfance [42]. Les voix sont alors comprises comme les messagers des problèmes de l'entendeur, pas comme des problèmes en soi. La présence de déclencheurs des voix bien repérables fait même partie des facteurs permettant de prédire en moyenne combien de temps dureront les voix [16, 17].

Des différences d'attitudes et de croyances purent être mises en évidence entre les groupes d'entendeurs de voix à répercussions positives et à répercussions négatives [41] : ainsi, les interprétations qui diminuaient le plus l'anxiété étaient psychodynamiques (par exemple, les voix étant compris comme des manifestations d'archétypes jungiens), mystiques (les voix témoignaient d'un développement spirituel, d'un élargissement de la conscience) et parapsychologiques (une sensibilité ou un don qu'il faut apprendre à contrôler). Les systèmes d'interprétations les moins efficaces sont ceux qui sortent les voix du champ de contrôle du sujet (par exemple, l’interprétation biomédicale ou démoniaque).

Des modèles psychologiques tentent de rendre compte de ce que le type d'interprétation ou d'appréciation (appraisal) des voix prédit mieux le niveau de détresse que, par exemple, la fréquence d'occurrence de ces voix [37] ou leur contenu, lequel ne diffère pas des hallucinations auditives de schizophrènes [38]. Ainsi, Garety et al. [19] proposait qu'une interprétation attribuant une origine externe aux voix provoquait plus d'anxiété qu'une attribution à une cause interne. Pour Brett et al. [11], c'est l'impersonnalisation plutôt que l'externalisation qui est la plus stressante : par exemple, une explication neurochimique des voix ne fait pas nécessairement 
sens pour l'individu, quand bien même il s'agit d'une cause interne. Alors que certaines interprétations des voix en termes de divinités ou d'esprits produisent souvent des arrangements réconfortants [46]. Ces recherches sur le type d'interprétation selon les axes interne/externe ou personnelle/impersonnelle donnent des indications directes sur les stratégies d'intervention du clinicien : rationalisation, normalisation, tolérance à la paranormalité, etc.

Hallucinations non-psychotiques chez l'enfant et l'adolescent

Il arrive aussi à des enfants et des adolescents d'entendre des voix, ce qui incite des cliniciens à se poser la question délicate d'un diagnostic différentiel des hallucinations et des délires à ces âges [2, 28]. La majorité des études citées jusqu'ici ont examiné la prévalence des hallucinations et des idées délirantes chez des adultes. La documentation de leur prévalence pour des catégories d'âge plus jeunes n'a débuté que récemment. Il a ainsi été observé que des enfants non-psychotiques peuvent manifester des hallucinations dès l'âge de cinq ans [28].

Deux raisons font que l'hallucination chez l'enfant et l'adolescent constitue un enjeu clinique important pour la pédopsychiatrie. Tout d'abord, la non-reconnaissance de l'hallucination comme symptôme peut être lourde de conséquence en raison des risques évolutifs, rares mais délétères, vers la schizophrénie infantile. Mais, d'un autre côté, tirer des conclusions trop hâtives peut conduire à un traitement des symptômes par des psychotropes. Ces médications dont les effets sont encore mal connus risquent non seulement de masquer l'évolution, mais encore d'être responsables d'un impact sur le développement psychique de l'enfant.

Les données du continuum psychotique accentuent cette idée que les expériences psychotiques ne sont pas spécifiques ou hautement prédictives de psychoses, puisqu'elles se produisent chez de nombreuses personnes sans psychopathologie [13]. Cela est particulièrement vrai pour les jeunes : par exemple, McGorry et al. [34] administrèrent un questionnaire sur les symptômes prodromiques de la schizophrénique à 657 lycéens et trouvèrent que près de la moitié d'entre eux répondaient aux critères de la phase prodromique de la schizophrénie. Néanmoins, il y a encore relativement peu d'études suivant l'évolution d'enfants rapportant des expériences psychotiques sans être hospitalisés $[16,17]$.

Les éléments de diagnostic différentiel attendus par les pédopsychiatres impliquent que soient mieux différenciés ce qui peut relever d'un «fonctionnement limite » de ce qui correspond à un «fonctionnement psychotique ». Les classifications actuelles occasionneraient trop d'erreurs dites de «faux positifs », c'est-à-dire d'enfants et d'adolescents qui ont relaté des hallucinations et sont traités pour psychose ou schizophrénie infantiles alors que les cliniciens les prenant en charge ne confirment pas ce diagnostic (par exemple, [50]).

Les interprétations du « continuum psychotique »

L'hypothèse d'un continuum psychotique implique qu'on observe les mêmes symptômes chez des patients ayant des troubles psychotiques diagnostiqués et dans la population générale. Plusieurs autres appellations ont tenté de rendre compte d'expressions de la psychose en-deçà de sa manifestation clinique : schizophrénie latente de Bleuler [8], psychoticisme chez Eysenck [7], enclin à la psychose (psychosis proneness), schizotypie ou états mentaux à risques [13, 15, 51]. Pourtant, faire l'expérience de délires ou d'hallucinations ne serait pas inévitablement associé avec la présence d'un trouble mental, de nombreux autres facteurs entrant en jeu. De plus, contrairement à ce qu'affirment certains auteurs ([55], p. 47), il n'est pas certain que des expériences dont les contenus sont similaires impliquent les mêmes mécanismes psychologiques. Ce serait méconnaître les travaux distinguant différents types de délires et d'hallucinations selon d'autres critères phénoménologiques et structuraux [29, 30].

Une distinction importante peut être faite entre expériences psychotiques, dont la prévalence est 
environ de $8 \%$ et symptômes psychotiques subcliniques, dont la prévalence est environ de $4 \%$ [56]. Les symptômes psychotiques subcliniques sont accompagnés de détresse et de démarches de recherche de soin, sans toutefois correspondre aux critères d'une psychose déclarée. Évidemment, cette distinction entre expériences et symptômes psychotiques dépend des critères utilisés pour repérer ce besoin de traitement. Les recherches mettent surtout l'accent sur différentes dimensions de la détresse associée à l'expérience et son influence sur le comportement [39, 47].

Le modèle de van Os et al. [56] fait des expériences psychotiques une possibilité commune et transitoire $\mathrm{du}$ fonctionnement mental, présente au cours du développement normal et particulièrement à l'adolescence. Parfois, ces expériences deviendraient persistantes et augmenteraient la possibilité de développer des troubles psychotiques sévères. Mais les transitions d'une forme de la psychose à une autre restent encore mal connues. Peu d'études précisent quelle fréquence, durée ou intensité des expériences psychotiques fait pencher un individu d'un côté à l'autre du continuum. Certains arguent qu'il n'y a pas une relation totalement linéaire mais qu'une accumulation de symptômes psychotiques atteindra un seuil pathologique [23]. Il n'a pas été établi ce qu'était ce seuil ou quels facteurs seraient à prendre en compte pour expliquer ce brusque changement. Il est révélateur que des notions comme celles de «prépsychose » en deviennent insaisissables [10].

\section{Problématisation}

Les interprétations du continuum psychotique que nous avons recensées proviennent surtout de travaux anglo-saxons qui prennent appui sur une classification des maladies mentales découlant - même si c'est par un retour critique - du DSM. La rencontre avec d'autres classifications et d'autres épistémologies n'est pas sans déclencher des vagues d'interrogations aux multiples réponses. La première concerne le mode d'évaluation par questionnaires de la psychose, la deuxième porte sur la théorie psychopathologique à laquelle sont renvoyées ces données.

\section{L'évaluation par questionnaires ou entretiens}

La volonté de fixer certaines idées ou expériences psychotiques dans des items pose question : comment peut-on justifier que certaines idées soient délirantes en soi ? Il n'y a qu'à constater l'usage bancal de cette notion indéfinissable d'idée délirante dans les derniers DSM, basé sur un rejet de la prise en considération de la dynamique du sujet, afin d'essayer de s'en tenir à ses comportements. Cette focalisation sur les aspects de surface et sur la pluralité des apparences imaginaires conduit au piège d'un socio-constructivisme, les analyses ne se dégageant pas suffisamment des contraintes de pensée, ou "prédicats anthropologiques », propres à chaque culture à un moment donné. Les cliniciens ont toujours eu à se méfier de ces appels à des références extérieures, hors des observations réalisées dans le cadre clinique.

De ce fait, les questionnaires mesurant les expériences psychotiques restent relativement mal conçus par rapport à leur objectif. Par exemple, la plupart de leurs items mélangent une expérience psychotique avec l'une de ses interprétations. L'inventaire des délires de Peters [39] a élargi les mesures au-delà du seul contenu de l'item en incluant des souséchelles de conviction, de préoccupation et de détresse permettant de rentrer davantage dans les détails de chaque croyance. Néanmoins, ces mesures restent secondaires.

A l'inverse, l'Appraisals of Anomalous Experiences Interview (AANEX) de Caroline Brett [11], en reprenant une méthodologie à base d'entretiens diagnostiques semi-structurés, a montré qu'il s'agissait toujours d'une procédure de qualité, ayant une meilleure validité et fiabilité que les mesures par questionnaire pour la détection de besoin de traitement chez des individus ayant des expériences psychotiques. La visée de l'AANEX est d'évaluer, par une série de questions ouvertes laissant davantage de place à une parole, la manière dont une personne apprécie et intègre subjectivement ses expériences psychotiques. Pour chaque expérience que le sujet reconnaît avoir vécu, des questions lui sont posées concernant le contexte de survenue, la fréquence... Les émotions positives et négatives associées à l'expérience sont explorées, ainsi que les interprétations, les cognitions, les comportements et les traits de personnalité par d'autres séries de questions. En approfondissant la phénoménologie de ces expériences tout en leur rendant leur forme singulière, ces questions apportent des précisions qui permettraient de mettre à jour des signes distinctifs pour un diagnostic différentiel plus précis. Ainsi, les entretiens diagnostiques 
obtiennent généralement moins de faux-positifs que les questionnaires auto-administrés, mais les deux seraient complémentaires ([56], p. 185). Or, Brett est actuellement l'une des seules à avoir réhabilité cette méthodologie pour ce type d'expériences, même si cela reste dans une approche de recherche en psychologie cognitive invitant à laisser plus de place à une expression personnelle pour ensuite la filtrer à travers une cotation n'autorisant pas l'imprévu.

Des expériences entre psychose et névrose

Il y a une inadéquation entre, d'un côté, les tableaux de la psychose déclarée et de la schizophrénie découlant du DSM et, de l'autre côté, les structures psychopathologiques de la névrose et de la psychose décrites par les psychanalystes et une certaine tradition clinique française. Cette inadéquation tient notamment au fait que des hallucinations et des délires ont pu être observés dès la fin du XIXe siècle sur des sujets non-psychotiques [25, 12]. Les notions de folie névrotique, d'hystérie crépusculaire, ou encore d'hallucinoses, englouties dans la description bleulérienne des schizophrénies [30], rendaient plus facilement compte de la prévalence des délires et hallucinations dans la population générale.

Quand bien même le paradigme des grandes structures psychopathologiques a fait l'objet de critiques pertinentes, il n'est pas interdit qu'il puisse être mis à profit dans les débats actuels. En précisant mieux les critères véritablement spécifiques au fonctionnement psychotique, le diagnostic différentiel des expériences psychotiques serait probablement amélioré [31, 32]. Ainsi, des observations cliniques qui ne sont pas que le fait de psychanalystes amènent à distinguer un système de manifestations névrotiques s'opposant au système décelé dans la psychose. Les différences se situent sur les types de discours, de dissociation, d'automatisme, de projection, d'angoisse, de croyance, de néologisme, de relation aux idéaux, d'influence du milieu, d'adresse, d'hypnotisabilité et de logique évolutive. Sans prétendre tout régler en quelques mots, voici quelques indications sur ces lieux de différenciation.

Il est caractéristique que le discours délirant du névrotique s'imprègne d'une signification phallique et d'une thématique sexuelle, tandis que les déformations du discours psychotique paraissent êtres dues à une dérive métonymique de la chaîne signifiante suscitée par un vide qu'elle ne peut dire. Si des manifestations de cette déstructuration se présentent dans le langage, il serait faux de croire qu'il n'y a de néologismes que psychotiques. En effet, bien des états confusionnels témoignent de la production de mots inventés, voire de langues inconnues, appelées glossolalies. Le caractère proprement psychotique du néologisme est d'être inséré passivement au sein de la langue maternelle pour suturer la chaîne signifiante désarrimée. Les néologismes névrotiques renvoient quant à eux à un référent précis, émergent d'une construction historique, comme ces images déformées du rêve condensant plusieurs significations chargées. Ce sont des néologismes actifs, des innovations imaginaires et non des tentatives pour remédier à une lacune du symbolique. Quand les mots semblent venir de l'extérieur par des hallucinations verbales, la même logique peut aider à établir une distinction. L'injure annihilante ("vache, putain, salope ») est caractéristique de l'hallucination verbale du psychotique : l'Autre y vise l'être de déchet du sujet comme source de sa jouissance. Cette exigence de jouissance reste tempérée chez le névrotique par la fonction paternelle, ce dont témoigne le caractère répressif des injonctions perçues, qui se présentent donc comme des reproches ou des interdits.

Ces différences dans le registre de la langue ne représentent qu'une partie des signes qu'une clinique inspirée par Lacan [27] nous amène à repérer. Nous les présentons surtout pour montrer que la manifestation élargie d'expériences réputées psychotiques n'implique pas nécessairement de prolonger aveuglément les modèles propres au fonctionnement psychotique.

Conclusion

Ceux que nous appelons psychose pourrait n'être que l'extrémité négative et rare d'une expérience courante. C'est ce que suggèrent les données épidémiologiques et psychométriques sur les expériences réputées psychotiques dans la population générale. Les facteurs étiologiques de la psychose seraient aussi à chercher dans ce qui fait que certaines expériences communes et transitoires, qui accompagnent généralement le développement psychologique, puissent persister et 
s'aggraver. Résoudre ces énigmes passe donc par un intérêt accru pour les expériences réputées psychotiques, leur détection précoce, leurs traitements et leurs transitions jusqu'à d'autres formes de psychose.

Malgré la richesse du débat sur ces données, on ne peut s'empêcher de se demander s'il ne repose pas sur un faux problème. Certains diront que la notion d' «expériences réputées psychotiques » est une extension injustifiée d'une définition bleulérienne de la psychose à des signes qui relèvent d'autres troubles. D'autres y verront les intérêts mercantiles d'une industrie pharmacologique qui fait alliance avec une «fonction psy » pour détecter une symptomatologie positive - hallucinations et délires - plus aisée à délimiter par rapport à une «norme » que les symptômes plus imprécis que l'on range dans la catégorie des «négatifs ». Il n'est pourtant pas impossible que ces expériences, d'abord mises en évidence par des parapsychologues, pointent de façon originale une faille du savoir psychopathologique. Comment obtenir les coordonnées exactes de la folie par rapport à la déraison, au religieux, au culturel, et également à cet objet intermédiaire qu'est le paranormal ? Celui-ci est officiellement exclu de la rationalité et néanmoins, comme le montrent les sondages, il est partagé par une portion importante de la population sous forme de croyances ou de formes données à des vécus. Les pistes d'hier et d'aujourd'hui semblent se diriger vers une réhabilitation des entretiens diagnostics approfondis ne se fixant pas sur l'évaluation de l'écart d'une expérience perturbante par rapport à une norme générale, mais en analysant le mode d'appréciation singulier d'un contenu qui ne saurait être posé comme délirant en soi. 
1 American Psychiatric Association. Diagnostic and statistical manual for mental disorders, 4th edition. Washington DC: American Psychiatric Association ; 1994.

2 Askenazy F, Dupuis G, Dor E, Lestideau K, Meynadier A, Myquel A. Clinique des hallucinations auditives chez l'enfant non-psychotique. Neuropsychiatrie de l'enfance et de l'adolescence $2009 ; 57: 25-31$.

3 Barrett TR, Etheridge JB. Verbal hallucinations in normals: I. People who hear voices. Applied Cognitive Psychology $1992 ; 6$ : 379-387.

4 Belz M. Aussergewöhnliche Erfahrungen. Göttingen: Hogrefe. «Fortschritte der Psychotherapie »;2009.

5 Bentall RP. The illusion of reality: A review and integration of psychological research on hallucinations. Psychological Bulletin 1990 ; 107 : 82-95.

6 Bentall RP, Slade PD. Reality testing and auditory hallucinations: A signal-detection analysis. British Journal of Clinical Psychology 1985 ; 24 : 159-169.

7 Berenbaum H, Boden MT, Baker JP, Dizen M, Thompson RT, Abramowitz A. Emotional correlates of the different dimensions of schizotypal personality disorder. Journal of Abnormal Psychology $2006 ; 115: 359-368$.

8 Bleuler E. Démence précoce ou le groupe des schizophrénies. Paris : Centre d'Etude Psychiatrique ; 1911/1926.

9 Bradbury DA, Stirling J, Cavill J, Parker A. Psychosis-like experiences in the general population: An exploratory factor analysis. Personality and Individual Differences 2009 ; 46(7) : 729-734.

10 Brémaud N. Prépsychose : revue de la littérature et approche critique. L'information psychiatrique $2008 ; 84(5)$ : 383-393.

11 Brett CMC, Peters EP, Johns LC, Tabraham P, Valmaggia LR, McGuire PK. Appraisals of Anomalous Experiences Interview (AANEX): a multidimensional measure of psychological responses to anomalies associated with psychosis. The British Journal of Psychiatry 2007 ; 191(51) : 23-30.

12 Breuer J, Freud S. Etudes sur l'hystérie. Paris : PUF «Bibliothèque de psychanalyse »; $1895 / 1973$.

13 Chapman LJ, Chapman JP, Kwapil TR, Eckblad M, Zinser MC. Putatively psychosis-prone subjects 10 years later. J Abnorm Psychol $1994 ; 103$ : 171-183.

14 Ciompi L. Is there really a schizophrenia?: The longterm course of psychotic phenomena. British Journal of Psychiatry $1984 ; 145: 636-640$.

15 Claridge G (ed). Schizotypy: Implications for illness and health. New York : Oxford University Press ; 1997.

16 Escher A, Romme M, Buiks A, Delespaul P, Van Os J. Independent course of childhood auditory hallucinations: a sequential 3-year follow-up study. British Journal of Psychiatry 2002a ; 181 Suppl. 43 : 10-18.

17 Escher A, Romme M, Buiks A, Delespaul P, Van Os J. Formation of delusional ideation in adolescents hearing voices: a prospective study. American Journal of Medical Genetics 2002b ; 114 : 913-920.

18 Freud S. L'interprétation des rêves. Paris : PUF «Grands ouvrages »; 1900/1999.

19 Garety PA, Kuipers E, Fowler D, Freeman D, Bebbington PE. A Cognitive Model of the Positive Symptoms of Psychosis. Psychological Medicine 2001 ; 31(02) : 189-195. 
20 Gauld A. The founders of psychical research. London: Routledge \& Kegan Paul ; 1968.

21 Gori R, Del Volgo MJ. Exilés de l'intime : Médecine et psychiatrie au service du nouvel ordre économique. Paris : Denoël « Médiations »; 2008.

22 Gurney E, Myers FWH, Podmore F. Les hallucinations télépathiques. Paris : Alcan, 1886/1891.

23 Häfner H. What is schizophrenia? European Archives of Psychiatry and Clinical Neuroscience $1988 ; 238(2): 63-72$.

24 Irwin HJ. The Psychology of Paranormal Belief : A Researcher's Handbook. Herfordshire: University of Hertfordshire Press ; 2009.

25 Janet P. L'automatisme psychologique. Essai de psychologie expérimentale sur les formes inférieures de l'activité humaine. Paris : Alcan ; 1889.

26 Johns LC, Cannon M, Singleton N, Murray RM, Farrell M, Brugha T, Bebbington P, Jenkins R, Meltzer H. Prevalence and correlates of self-reported psychotic symptoms in the British population. British Journal of Psychiatry 2004 ; 185(4) : 298-305.

27 Lacan J. Le Séminaire, Livre III : Les Psychoses (1955-1956). Paris : Seuil « Champ freudien »; 1981.

28 Larøi F, Van der Linden M, Goëb JL. Hallucinations et idées délirantes chez les enfants et adolescents : mise en perspective avec les travaux réalisés chez l'adulte. Neuropsychiatrie de l'enfance et de l'adolescence 2009 ; 57 : 32-37.

29 Le Maléfan P. L'hallucination télépathique ou véridique dans la psychopathologie de la fin du XIXe siècle et du début du XXe siècle. L'Evolution Psychiatrique 2008 ; 73(1) : 15-40.

30 Maleval JC. Folies hystériques et psychoses dissociatives. Paris : Payot ; 1981.

31 Maleval JC. Logique du délire. Paris : Masson « Médecine et psychothérapie »; 1996.

32 Maleval JC. La forclusion du Nom-du-Père. Le concept et sa clinique. Paris : Seuil « Champ freudien »; 2000.

33 McCreery C, Claridge G. Healthy schizotypy: the case of out-of-the-body experiences. Personality and Individual Differences 2002 ; 32(1) : 141-154.

34 McGorry PD, McFarlane C, Patton GC, Bell R, Hibbert ME, Jackson HJ et al. The prevalence of prodromal features of schizophrenia in adolescence: a preliminary survey. Acta Psychiatrica Scandinavica 1995 ; 92(4) : 241-249.

35 McKellar P. Experience and behaviour. London: Penguin ; 1968.

36 Miller LJ, O'Connor E, DePasquale T. Patients' attitudes to hallucinations. American Journal of Psychiatry $1993 ; 150: 584-588$.

37 Morrison AP, Baker CA. Intrusive thoughts and auditory hallucinations: a comparative study of intrusions in psychosis. Behaviour Research and Therapy 2000 ; 38(11) : 1097-1106.

38 Pennings $\mathrm{M}$, Romme M. Stemmen horen bij schizofrenie patienten, patienten met een dissociatieve stoornis en bij niet patienten. In: de Hert M, Thijs E, Peuskens I, Petri D, van Raay B, Zin in waanzin: De wereld van schizofrenie. Antwerp: EPO, 1996 : 127-140.

39 Peters ER, Joseph SA, Garety PA. Measurement of delusional ideation in the normal population : introducing the PDI (Peters et al. Delusions Inventory). Schizophrenia Bulletin 1999 ; 25 : $553-576$.

40 Posey TB, Losch ME. Auditory hallucinations of hearing voices in 375 normal subjects. Imagination, Cognition and Personality $1983 ; 2: 99-113$.

41 Romme M, Escher A. Hearing voices. Schizophrenia Bulletin 1987 ; 15(2) : 209-216.

42 Romme M, Escher A. Making Sense of Voices. Mind. London ; 2000. 
43 Romme M, Escher A. Empowering people who hear voices. In: Haddock G, Slade PD, Cognitive behavioural interventions with psychotic disorders. London: Routledge, 1996 : 137 150.

44 Sarbin TR, Juhasz JB. The historical background of the concept of hallucination. Journal of the History of the Behavioral Sciences 1967 ; 5 : 339-358.

45 Schneider K. Psychopathologie clinique. Paris : Nauwelaerts ; 1949/1970.

46 Schofield K, Claridge G. Paranormal experiences and mental health: Schizotypy as an underlying factor. Personality and Individual Differences 2007 ; 43(7) : 1908-1916.

47 Serper M, Dill CA, Chang N, Kot T, Elliot J. Factorial structure of the hallucinatory experience : continuity of experience in psychotic and normal individuals. Journal of Nervous and Mental Disease $2005 ; 193: 265-272$.

48 Sidgwick H, Johnson A, Myers FWH, Podmore F, Sidgwick EM. Report on the census of hallucinations. Proceedings of the Society for Psychical Research 1894 : 10 : 25-422.

49 Slade PD, Bentall RP. Sensory deception: A scientific analysis of hallucination. London: Croom-Helm ; 1988.

50 Stayer C, Sporn A, Gogtay N, Tossel J, Lenane M, Gochman P et al. Looking for childhood schizophrenia: case series of false positives. Journal of American Academy of Child and Adolescence Psychiatry 2004 ; 43(8) : 1026-1029.

51 Stefanis N, Hanssen M, Smirnis N, Avramopoulos D, Evdokimidis I, Stefanis C, Verdoux H, Van Os J. Evidence that three dimensions of psychosis have a distribution in the general population. Psychological Medicine 2002 ; 32(2) : 347-358.

52 Tien AY. Distribution of hallucinations in the population. Social Psychiatry and Psychiatric Epidemiology $1991 ; 26$ : 287-292.

53 Van Os J, Burns T, Cavallaro R, Leucht S, Peuskens J, Helldin L et al. Standardized remission criteria in schizophrenia. Acta Psychiatrica Scandinavica 2006 ; 113 : 91-95.

54 Van Os J, Delespaul, P. Toward a world consensus on prevention of schizophrenia. Dialogues in Clinical Neuroscience $2005 ; 7(1)$ : 53-67.

55 Van Os J, Hanssen M, Bak I, Myin-Germeys M, Marcelis R, Bij1 W, Vollebergh P, Delespaul P. Cognitive epidemiology: Psychological and social risk mechanisms for psychosis. In: Häfner H, Risk and Protective Factors in Schizophrenia: Towards a Conceptual Model of the Disease Process. Darmstadt, Netherlands: Steinkopf, 2002 : 39-60.

56 Van Os J, Linscott R, Myin-Germeys I, Delespaul P, Krabbendam L. A Systematic Review and Meta-Analysis of the Psychosis Continuum: Evidence for a Psychosis Pronenes - persistence - impairment Model of Psychotic Disorder. Psychological Medicine 2009 ; 39(02) : 179-195.

57 Verdoux H, van Os J. Psychotic symptoms in non-clinical populations and the continuum of psychosis. Schizophrenia Research 2002 ; 54(1-2) : 59-65.

Pas de conflit d'intérêts. 